\title{
Two-species cold atomic beam
}

\author{
Nathan Lundblad,* David C. Aveline, Robert J. Thompson, James M. Kohel, Jaime Ramirez-Serrano, \\ William M. Klipstein, Daphna G. Enzer, Nan Yu, and Lute Maleki \\ Jet Propulsion Laboratory, California Institute of Technology, 4800 Oak Grove Drive, \\ Pasadena, California 91109-8099
}

Received February 27, 2003; revised manuscript received August 8, 2003; accepted September 2, 2003

\begin{abstract}
We generate a bright atomic beam containing laser-cooled rubidium and cesium, and we use this beam to load a mixed-species ultrahigh-vacuum (UHV) magneto-optical trap. We have characterized our two-species atomic beam over a range of operating conditions, and we obtain similar atom fluxes for each species. Within the UHV trap, interspecies inelastic collisions are observed in the form of enhanced decay rates of a given species in the presence of a second trapped species. We analyze the trap decays to obtain a loss rate due to heteronuclear cold collisions, and we compare our result to similar measurements in vapor-cell traps [Phys. Rev. A 63, 033406 (2001)]. (C) 2004 Optical Society of America OCIS codes: $140.3320,020.7010,020.2070$.
\end{abstract}

\section{INTRODUCTION}

In recent years there has been increasing interest in the physics of ultracold gases composed of two or more distinct atomic species. Cold collisions in mixed-species magneto-optical traps (MOTs) have been studied for a number of alkali gases. ${ }^{1-8}$ Sympathetic cooling in twospecies magnetic traps has allowed exploration of quantum degeneracy in a number of fermionic species, ${ }^{9-11}$ as well as studies of mixtures of quantum degenerate fluids. ${ }^{12,13}$ In addition, colocating cold atomic samples of two species under nearly identical conditions is desirable for a number of precision measurements, including electric dipole moment searches ${ }^{14}$ and tests of local Lorentz invariance $^{15}$ and the equivalence principle.

Many of these experiments could be enhanced with a bright two-species cold atomic beam (CAB) source. For studies of quantum degenerate systems, such an apparatus is useful simply as a beam source of cold atoms, allowing high numbers of atoms to be trapped under ultrahigh vacuum (UHV) with a relatively compact apparatus. For studies of heteronuclear cold collisions in a UHV MOT, such a source offers capabilities that are not possible in a vapor-cell apparatus, which all other investigations to date have employed. Most importantly, the two-species beam allows the loading of each individual species to be independently controlled. Furthermore, as studies can be carried out in a UHV environment, the effects of background collisions can be effectively neglected. A bright two-species cold atomic beam would also open the door to studies of light-assisted cold heteronuclear collisions within the beam itself. Such an experiment allows one to control both the polarization of the light and the orientation of the atoms with respect to the collision axis, information that cannot be obtained from experiments conducted within a MOT. ${ }^{16}$

In this paper, we present a novel and simple twospecies $\left({ }^{133} \mathrm{Cs}+{ }^{87} \mathrm{Rb}\right)$ atomic beam source, and we utilize this beam source to perform a preliminary study of cold collisions in a separate mixed-species trap under UHV conditions. The present experiment allows independent control of the cesium and rubidium traps; in this way we observe trap loading and decay rates for each species with and without the presence of the second species. Simple modeling of these observed differences allows measurements of the heteronuclear cold collision rate, which we compare to results from recent experiments in vapor-cell traps. ${ }^{7}$

\section{EXPERIMENTAL DESCRIPTION}

Our cold atomic beam is generated with a modified pyramidal $\mathrm{MOT}^{17}$; this apparatus has been described in a recent report. ${ }^{18}$ In brief, our pyramidal trap consists of a large four-sided pyramidal mirror assembly that is truncated just before the apex. At the truncated apex, a separate retro-optic (consisting of a $\lambda / 4$ plate with a highreflectance gold coating on the second surface) contains a 1-mm aperture. A slow beam of cold atoms is extracted through this aperture via the resulting radiation pressure imbalance. ${ }^{19}$ A detailed characterization of the singlespecies cesium CAB can be found in Ref. 18 .

To operate our pyramidal trap as a two-species CAB source, four optical frequencies are required for laser trapping and cooling the two alkali species, as illustrated in Fig. 1. The $\mathrm{Cs}$ and $\mathrm{Rb}$ trapping lasers are combined on a nonpolarizing beam splitter (NBS). One-half the power in each beam is directed to the pyramidal MOT, and a portion of the remaining half is directed to a UHV ( $P_{\text {tot }}$ $<10^{-10}$ Torr) MOT located $36 \mathrm{~cm}$ downstream from the center of the pyramidal trap in an uncoated glass cuvette. Circular polarization is approximated in the 852-nm and 780-nm trapping lasers by sending the linearly polarized beams through a single wave plate (QWP). This wave plate, with a nominal retardance of $\lambda / 4$ at $850 \mathrm{~nm}$, is rotated to simultaneously optimize the flux in the Cs and $\mathrm{Rb}$ atomic beams. Similarly, a single wave plate creates near-circular polarization in the overlapped trap ping beams for the UHV MOT. The combined beams for the pyramidal MOT are expanded by a factor of 30 to ap- 


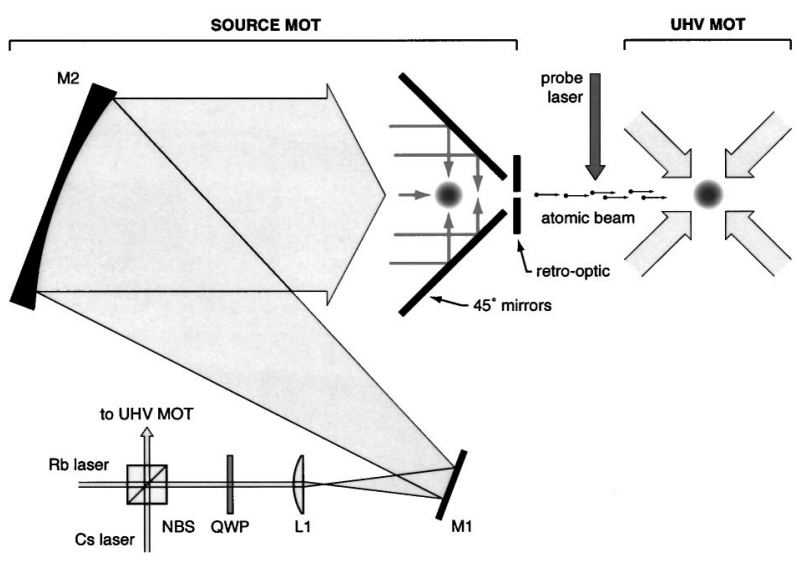

Fig. 1. Schematic diagram of the experimental apparatus, including the two-species cold atomic beam source and UHV MOT. The distance from the source to the UHV MOT is $36 \mathrm{~cm}$.

proximately $15 \mathrm{~cm}$ in diameter and aligned into the pyramidal trap along the longitudinal $(z)$ axis.

The lasers used for the two-species traps include a Ti:sapphire ring laser and three diode-laser systems. The trapping light for ${ }^{87} \mathrm{Rb}$ is generated by the Ti:sapphire laser, which delivers up to $200 \mathrm{~mW}$ of power at 780 $\mathrm{nm}$ to each of the two Rb traps. The repumping frequency for $\mathrm{Rb}$ is provided by an external-cavity diode laser (ECDL) that produces up to $15 \mathrm{~mW}$ at $780 \mathrm{~nm}$. The trapping frequency for $\mathrm{Cs}$ is generated via an ECDL, which is further amplified by a tapered-cavity diode amplifier. The amplified output is coupled to the experiment via a polarization-maintaining optical fiber, so that the total Cs trapping power available to the experiment is $300-350 \mathrm{~mW}$ at $852 \mathrm{~nm}$. Another ECDL serves as the Cs repumping laser. The $\mathrm{Rb}$ repumping laser is combined with the trapping lasers, while the Cs repumping laser is delivered separately via optical fibers to the source and UHV trap regions. By shuttering the repumping beams to either trap, the loading of the Cs source and UHV MOTs can be independently controlled.

\section{CHARACTERIZING THE DUAL-BEAM SOURCE}

We detect the atomic beams via resonance fluorescence detection with a pair of overlapping frequency-modulated

Table 1. Typical Parameters for the Cold Atomic Beam (CAB) Source and UHV MOT

\begin{tabular}{|c|c|c|c|c|c|}
\hline & \multicolumn{2}{|c|}{ CAB Source } & \multicolumn{2}{|c|}{ UHV MOT } & \multirow[b]{2}{*}{ Notes } \\
\hline & Cs & ${ }^{87} \mathrm{Rb}$ & Cs & ${ }^{87} \mathrm{Rb}$ & \\
\hline$I_{0} / I_{\mathrm{sat}}$ & 0.9 & 0.7 & 18 & 15 & $a$ \\
\hline$\Delta / \Gamma$ & -2.1 & -2.3 & -2.1 & -2.3 & $b$ \\
\hline $\mathfrak{F}($ atoms $/ \mathrm{s})$ & $2 \times 10^{8}$ & $1 \times 10^{8}$ & $\ldots$ & $\ldots$ & $c$ \\
\hline$N$ (atoms) & $\ldots$ & $\ldots$ & $6 \times 10^{8}$ & $2 \times 10^{8}$ & \\
\hline
\end{tabular}

${ }^{a}$ The laser intensities $I_{0}$ are specified for each trapping beam, so that the total laser intensity incident on atoms in the UHV MOT is given by $I_{\text {tot }}=6 I_{0}$. These intensities are normalized by $I_{\text {sat }}$, the saturation intensity of the cycling transitions, where $I_{\text {sat }}=1.1 \mathrm{~mW} / \mathrm{cm}^{2}\left(1.7 \mathrm{~mW} / \mathrm{cm}^{2}\right)$ for $\mathrm{Cs}\left({ }^{87} \mathrm{Rb}\right)$.

${ }^{b}$ The laser detunings $\Delta$ are normalized by the natural linewidths $\Gamma$, where $\Gamma=5.2 \mathrm{MHz}(6.1 \mathrm{MHz})$ for $\mathrm{Cs}\left({ }^{87} \mathrm{Rb}\right)$.

${ }^{c}$ The atom flux $\mathfrak{F}$ is a lower bound derived from the UHV MOT loading rates and assuming a unit capture efficiency. probe beams tuned to the ${ }^{87} \mathrm{Rb} 5 S_{1 / 2}, F=2 \rightarrow 5 P_{3 / 2}$, $F^{\prime}=3$ and Cs $6 S_{1 / 2}, F=4 \rightarrow 6 P_{3 / 2}, F^{\prime}=5$ transitions A detuning of $-11 \mathrm{MHz} \quad\left(-2.1 \Gamma_{\mathrm{Cs}}\right.$, where $\Gamma_{\mathrm{Cs}}$ $=5.2 \mathrm{MHz}$ ) for the Cs trapping laser was found to optimize the flux of the $\mathrm{Cs}$ beam; for $\mathrm{Rb}$, the optimum detuning was found at $\Delta \approx-14 \mathrm{MHz}\left(-2.3 \Gamma_{\mathrm{Rb}}\right.$, where $\Gamma_{\mathrm{Rb}}$ $=6.1 \mathrm{MHz}$ ). A magnetic-field gradient of approximately $3 \mathrm{G} / \mathrm{cm}$ was found to simultaneously optimize the flux of the $\mathrm{Cs}$ and $\mathrm{Rb}$ atomic beams. By varying the angle of the probe with respect to the atomic beams, we determine the mean velocity of each atomic species from the resulting Doppler shift. The mean velocity of the $\mathrm{Rb}$ beam was measured to be $10 \mathrm{~m} / \mathrm{s}$ for typical operating conditions (cf. Table 1); the Cs beam velocity, measured at a much higher laser intensity $\left(I=3 I_{\text {sat }}\right.$ ), was $15 \mathrm{~m} / \mathrm{s}^{18}$ The divergence of the $\mathrm{CAB}$ is geometrically limited, and this divergence was previously measured to be $15 \mathrm{mrad}$ for the $\mathrm{Cs}$ beam in the current apparatus. ${ }^{18}$

The UHV MOT was characterized by employing two calibrated CCD cameras with narrow-band optical filters to selectively image the trapped $\mathrm{Cs}$ and $\mathrm{Rb}$ atom clouds. The calibrated imaging system allows us to determine the atom number as well as the loading and decay rates for the UHV trap. Our reported values for the atom flux (cf. Table 1) are determined from the loading rates observed in the UHV MOT and assuming a capture efficiency of unity $^{20}$; these values should be taken as lower bounds on the atomic beam flux. We note, however, that the fluxes reported here are still an order of magnitude lower than what we have measured previously in the single-species Cs CAB generated in this same apparatus. ${ }^{18}$ Much of this difference can be attributed to the lower optical powers (one third to one fourth) available to the pyramidal source MOT in the present study. This dual-species beam performance could be enhanced, however, by simply employing the appropriate dichroic beam splitter and wave plates to allow a greater fraction of the available laser powers at $780 \mathrm{~nm}$ and $852 \mathrm{~nm}$ to be simultaneously directed to the source MOT. The current experimental parameters and performance figures for the CAB and UHV MOT are summarized in Table 1.

Typical loading curves for the Rb and Cs UHV MOTs are shown in Fig. 2, where both species are present in the $\mathrm{CAB}$ but not in the UHV MOT. Here we are suddenly switching $(<2 \mathrm{~ms})$ on the repumper light for one species in both the source and UHV MOT regions, allowing us to study the dynamics of the combined system of source MOT, propagating beam, and UHV MOT. The gradual onset of the MOT loading curves in Fig. 2 results from the time of formation of the $\mathrm{CAB}$, including the time of flight of atoms from the source to the UHV trap. We attribute the faster onset for loading into the Cs UHV MOT to the higher Cs vapor pressure in the source region, and thus a faster time of formation for the Cs beam.

\section{STUDYING HETERONUCLEAR COLD COLLISIONS WITH THE TWO-SPECIES BEAM}

We observe no measurable change in the flux of the $R b$ beam when the $\mathrm{Cs}$ beam is present, and vice versa; from this we conclude that heteronuclear cold collisions are not 


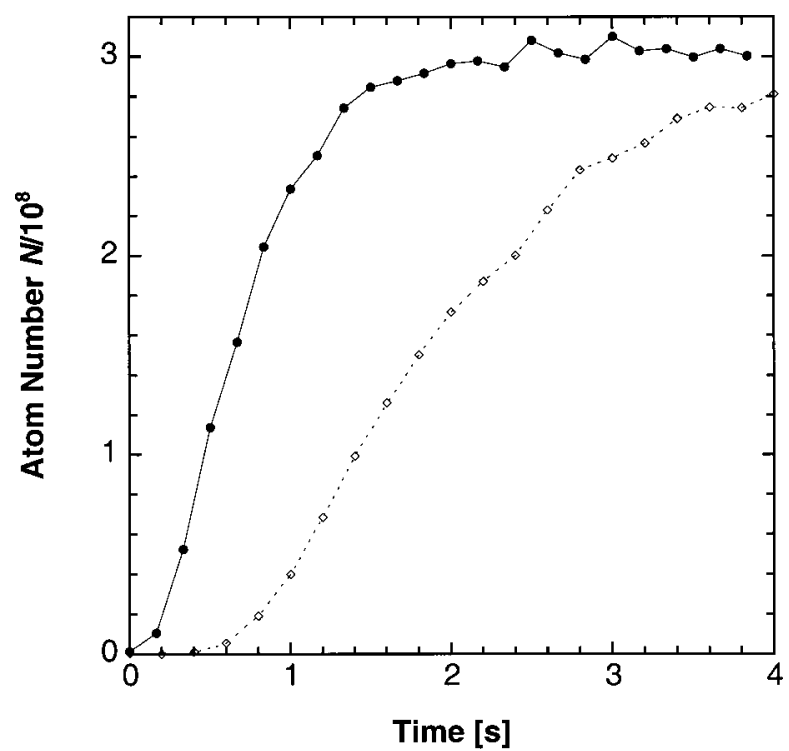

Fig. 2. Loading curves for the $\mathrm{Cs}$ (dots) and $\mathrm{Rb}$ (diamonds) UHV traps after switching on the cold atomic beam source.

a significant loss mechanism in the pyramidal trap or within the freely propagating beam. This is not unexpected, as the loss rate due to trapped atoms being extracted into the $\mathrm{CAB}$ dominates over all other loss mechanisms within the pyramidal MOT, and the densities within the atomic beam $\left(<2 \times 10^{7}\right.$ atom $\left./ \mathrm{cm}^{3}\right)$ are still an order of magnitude too low for these cold collisions to be observed. ${ }^{16,21,22}$

The effect of heteronuclear cold collisions is readily apparent within the mixed-species UHV MOT, however. We observe decreased trap lifetimes for the Cs MOT in the presence of simultaneously trapped $\mathrm{Rb}$, and vice versa. This behavior is illustrated in Fig. 3. From analysis of the observed Cs MOT decay rates with and without the cold $\mathrm{Rb}$ background, we obtain a measure of the loss rate due to heteronuclear cold collisions as detailed below.

Following the approach of Telles et al. ${ }^{7}$ we describe the general time dependence of our Cs UHV MOT using the rate equation ${ }^{16}$

$$
\frac{\mathrm{d} N_{\mathrm{Cs}}}{\mathrm{d} t}=L-\gamma N_{\mathrm{Cs}}-\beta \int_{\mathrm{V}} n_{\mathrm{Cs}}^{2} \mathrm{~d} \mathbf{r}-\beta^{\prime} \int_{\mathrm{V}} n_{\mathrm{Cs}} n_{\mathrm{Rb}} \mathrm{d} \mathbf{r}
$$

where $L$ is the loading rate (from the atomic beam), $\gamma$ is the loss rate due to collisions between the trapped cesium atoms and the thermal background gas, $\beta$ is the loss rate due to cold collisions among trapped cesium atoms, $\beta^{\prime}$ is the cesium loss rate due to cold collisions with trapped rubidium atoms, $N_{\mathrm{Cs}}$ is the number of trapped cesium atoms, and $n_{\mathrm{Cs}}$ and $n_{\mathrm{Rb}}$ are the density profiles of cesium and rubidium atoms in the UHV MOT. We observe the decay of the Cs MOT by switching off the Cs loading beam ( $L \rightarrow 0$ ) while leaving the Rb beam and MOT in steady state. All of our data were taken in the density-limited regime, where both $n_{\mathrm{Rb}}$ and $n_{\mathrm{Cs}}$ are constant within the trap. In this regime, Eq. (1) can be expressed as the decay rate equation

$$
\frac{\mathrm{d} N_{\mathrm{Cs}}}{\mathrm{d} t}=-\left(\beta n_{\mathrm{Cs}}+\beta^{\prime} n_{\mathrm{Rb}} F\right) N_{\mathrm{Cs}},
$$

where the loading rate $L$ is zero and we have also taken the loss rate $\gamma$ to be zero for the UHV trap. The factor $F$ represents the relative overlap of the $\mathrm{Rb}$ and $\mathrm{Cs}$ trapped atoms; from fluorescence images of the UHV MOT, we estimate $F \approx 0.8$ immediately after Cs loading is switched off, and this factor approaches unity within $0.5 \mathrm{~s}$ of decay. We fit the MOT decay curves with single exponential functions, as in Fig. 3. The initial points in which the overlap $F$ between the two trapped species may be varying are not included in fits to the "two-species" decays (although these points typically affect the result by less than one percent). From fits to the single-species Cs MOT decays and using the measured trap density $n_{\mathrm{Cs}}$, we determine a collisional loss rate $\beta=5 \times 10^{-11} \mathrm{~cm}^{3} / \mathrm{s}$. This result is consistent with previous work, ${ }^{23}$ although we note that the current measurement is at a significantly higher laser intensity. The cross-species collisional loss rate $\beta^{\prime}$ is determined from the difference in decay rates for the Cs MOT with and without the cold Rb atom background, yielding a value of

$$
\beta_{\mathrm{Cs}-\mathrm{Rb}}^{\prime}=\eta(0.7 \pm 0.2) \times 10^{-11} \mathrm{~cm}^{3} / \mathrm{s}
$$

where the uncertainty results from the scatter in the single exponential fits to six pairs of decays (each similar to those in Fig. 3), and the factor $\eta \equiv 1.0 \pm 0.2$ represents the error due to uncertainties in the atomic densities. This loss rate is comparable to the rates measured by Telles et $a l .{ }^{7}$ for Rb trap losses due to collisions with cold Cs atoms $\left(\beta_{\mathrm{Rb}-\mathrm{Cs}}^{\prime}\right)$ at similar laser intensities, although, as noted by these authors, there is no a priori reason to expect the reciprocal loss rates to be equal. ${ }^{24}$

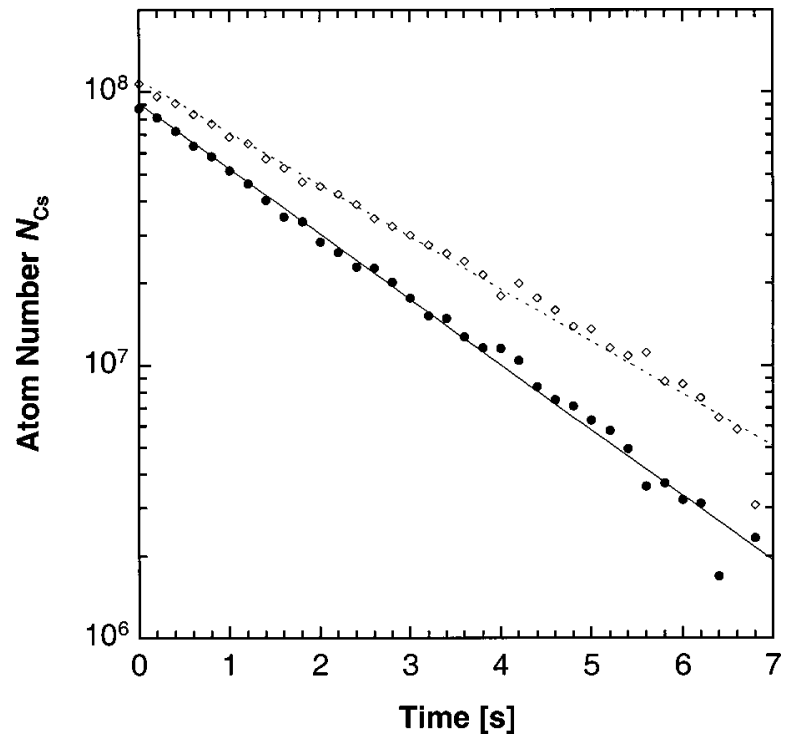

Fig. 3. Typical decay of the Cs UHV MOT with (dots) and without (diamonds) the presence of cold $\mathrm{Rb}$ atoms. The solid and dotted curves are least-squares fits of the data to single exponential decays with and without the cold Rb background, respectively. The fit to the "two-species" decay (solid curve) omits the first three data points, as discussed in the text. 


\section{SUMMARY AND CONCLUSIONS}

To our best knowledge, this is the first demonstration of a two-species continuous cold atomic beam based on threedimensional laser cooling. Recently, a high-flux twospecies Zeeman-slowed atomic beam was demonstrated by Hadzibabic et al. ${ }^{12}$ The relative advantages and disadvantages of MOT-based atomic beam sources versus other beam sources have been discussed previously. ${ }^{18,19}$

We have utilized our two-species beam to load a mixedspecies UHV MOT, and cross-species interaction effects were observed via the measured decay rates in the UHV trap. The current experiment allows independent control of the loading and decay of each species within the UHV trap, and the effects of cold collisions in the UHV MOT are isolated from the usual losses due to thermal gas backgrounds. These initial measurements hint at the broad utility of this two-species atomic beam plus UHV MOT system in the nascent field of heteronuclear cold-collision studies.

This simple and robust two-species atomic beam source may also prove useful in future experiments requiring comparison between two or more atomic species, including a proposed test of the equivalence principle that has been selected for development as a future National Aeronautics and Space Administration Microgravity Research Program flight experiment. ${ }^{25}$ Further work is underway to optimize performance of this source while minimizing the size and power requirements for flight applications.

\section{ACKNOWLEDGMENTS}

We would like to acknowledge helpful discussions with Eric Burt. This research was carried out at the Jet Propulsion Laboratory, California Institute of Technology, under a contract with the National Aeronautics and Space Administration, and was supported by the Jet Propulsion Laboratory Director's Research and Development Fund. One of the authors (N. Lundblad) is partially supported by a California Institute of Technology Robert Andrews Millikan Graduate Fellowship.

*Also at Department of Physics, California Institute of Technology, Pasadena, California 91125. Electronic address: lundblad@caltech.edu.

\section{REFERENCES AND NOTES}

1. M. S. Santos, P. Nussenzveig, L. G. Marcassa, K. Helmerson, J. Flemming, S. C. Zilio, and V. S. Bagnato, "Simultaneous trapping of two different atomic species in a vaporcell magneto-optical trap," Phys. Rev. A 52, R4340-R4343 (1995).

2. G. D. Telles, L. G. Marcassa, S. R. Muniz, S. G. Miranda, A. Antunes, C. Westbrook, and V. S. Bagnato, "Inelastic cold collisions of a $\mathrm{Na} / \mathrm{Rb}$ mixture in a magneto-optical trap," Phys. Rev. A 59, R23-R26 (1999).

3. J. P. Shaffer, W. Chalupczak, and N. P. Bigelow, "Trap loss in a two-species $\mathrm{Na}-\mathrm{Cs}$ magneto-optical trap: Intramultiplet mixing in heteronuclear ultracold collisions," Phys. Rev. A 60, R3365-R3368 (1999).

4. U. Schlöder, H. Engler, U. Schünemann, R. Grimm, and M. Weidemüller, "Cold inelastic collisions between lithium and cesium in a two-species magneto-optical trap," Eur. Phys. J. D 7, 331-340 (1999).

5. Y. E. Young, R. Ejnisman, J. P. Shaffer, and N. P. Bigelow,
"Heteronuclear hyperfine-state-changing cold collisions," Phys. Rev. A 62, 055403 (2000).

6. L. G. Marcassa, G. D. Telles, S. R. Muniz, and V. S. Bagnato, "Collisional losses in a K-Rb cold mixture," Phys. Rev. A 63, 013413 (2001).

7. G. D. Telles, W. Garcia, L. G. Marcassa, V. S. Bagnato, D. Ciampini, M. Fazzi, J. H. Müller, D. Wilkowski, and E. Arimondo, "Trap loss in a two-species Rb-Cs magneto-optical trap,” Phys. Rev. A 63, 033406 (2001).

8. J. Goldwin, S. B. Papp, B. DeMarco, and D. S. Jin, "Twospecies magneto-optical trap with ${ }^{40} \mathrm{~K}$ and ${ }^{87} \mathrm{Rb}$," Phys. Rev. A 65, 021402(R) (2002).

9. A. G. Truscott, K. E. Strecker, W. I. McAlexander, G. B. Partridge, and R. G. Hulet, "Observation of Fermi pressure in a gas of trapped atoms," Science 291, 2570-2572 (2001).

10. B. DeMarco and D. S. Jin, "Onset of Fermi degeneracy in a trapped atomic gas,” Science 285, 1703-1706 (1999).

11. R. Onofrio and C. Presilla, "Reaching Fermi degeneracy in two-species optical dipole traps," Phys. Rev. Lett. 89, 100401 (2002).

12. Z. Hadzibabic, C. A. Stan, K. Dieckmann, S. Gupta, M. W. Zwierlein, A. Görlitz, and W. Ketterle, "Two-species mixture of quantum degenerate Bose and Fermi gases," Phys. Rev. Lett. 88, 160401 (2002).

13. G. Modugno, G. Roati, F. Riboli, F. Ferlaino, R. J. Brecha, and M. Inguscio, "Collapse of a degenerate Fermi gas," Science 297, 2240-2243 (2002).

14. B. C. Regan, E. D. Commins, C. J. Schmidt, and D. DeMille, "New limit on the electron electric dipole moment," Phys. Rev. Lett. 88, 071805 (2002).

15. D. Bear, R. E. Stoner, R. L. Walsworth, V. A. Kostelecký, and C. D. Lane, "Limit on Lorentz and CPT violation of the neutron using a two-species noble-gas maser," Phys. Rev. Lett. 85, 5038-5041 (2000).

16. J. Weiner, V. S. Bagnato, S. Zilio, and P. S. Julienne, "Experiments and theory in cold and ultracold collisions," Rev. Mod. Phys. 71, 1-85 (1999).

17. K. I. Lee, J. A. Kim, H. R. Noh, and W. Jhe, "Single beam atom trap in a pyramidal and conical hollow mirror," Opt. Lett. 21, 1177-1179 (1996).

18. J. Kohel, J. Ramirez-Serrano, R. J. Thompson, L. Maleki, J. L. Bliss, and K. G. Libbrecht, "Generation of an intense cold atom beam from a pyramidal magneto-optical trap: Experiment and simulation," J. Opt. Soc. Am. B 20, 1161-1168 (2003).

19. Z. T. Lu, K. L. Corwin, M. J. Renn, M. H. Anderson, E. A. Cornell, and C. E. Wieman, "Low-velocity intense source of atoms from a magneto-optical trap," Phys. Rev. Lett. 77, 3331-3334 (1996).

20. Detailed Monte-Carlo simulations of the CAB-loaded UHV MOT predict that the capture efficiency of cold atoms from the atomic beam by the UHV MOT is approximately 70 percent; this efficiency is limited primarily by the small $(1 \mathrm{~cm})$ diameter for the trapping laser beams.

21. J. Ramirez-Serrano, W. DeGraffenreid, and J. Weiner, "Polarization-dependent spectra in the photoassociative ionization of cold atoms in a bright sodium beam," Phys. Rev. A 65, 052719 (2002).

22. J. Ramirez-Serrano, W. DeGraffenreid, J. Weiner, E. Tiesinga, and P. Julienne, "Beamloss spectroscopy of cold collisions in a bright sodium beam," Phys. Rev. A (to be published).

23. D. Sesko, T. Walker, C. Monroe, A. Gallagher, and C. Wieman, "Collisional losses from a light-force atom trap," Phys. Rev. Lett. 63, 961-964 (1989).

24. While the primary focus of Ref. 7 was the study of Rb trap losses from cold collisions with $\mathrm{Cs}$, the authors mention that they also measured a value for $\beta_{\mathrm{Cs}-\mathrm{Rb}}^{\prime}$ that was twenty times lower than the corresponding value for $\beta_{\mathrm{Rb}-\mathrm{Cs}}^{\prime}$. We are uncertain as to whether this result is actually inconsistent with ours, as the trap parameters were not specified for their measurement.

25. See http://funphysics.jpl.nasa.gov/technical/library/pilist.html. 